\title{
LBB Stability of a Mixed Galerkin Finite Element Pair for Fluid Flow Simulations
}

\author{
Colin J. Cotter ${ }^{\mathrm{a}, *}$ David A. Ham ${ }^{\mathrm{b}}$ Christopher C. Pain ${ }^{\mathrm{b}}$ \\ Sebastian Reich ${ }^{\mathrm{c}}$ \\ ${ }^{a}$ Department of Aeronautics, Imperial College London, London SW7 2AZ, United \\ Kingdom \\ ${ }^{\mathrm{b}}$ Department of Earth Science and Engineering, Imperial College London, London \\ $S W^{\text {r }}$ 2AZ, United Kingdom \\ ${ }^{\mathrm{c}}$ Institut für Mathematik, Universität Potsdam, Am Neuen Palais 10, D-14469, \\ Potsdam, Germany
}

\begin{abstract}
We introduce a new mixed finite element for solving the 2- and 3-dimensional wave equations and equations of incompressible flow. The element, which we refer to as $\mathrm{P} 1_{\mathrm{D}}-\mathrm{P} 2$, uses discontinuous piecewise linear functions for velocity and continuous piecewise quadratic functions for pressure. The aim of introducing the mixed formulation is to produce a new flexible element choice for triangular and tetrahedral meshes which satisfies the LBB stability condition and hence has no spurious zeroenergy modes. The advantage of this particular element choice is that the mass matrix for velocity is block diagonal so it can be trivially inverted; it also allows the order of the pressure to be increased to quadratic whilst maintaining LBB stability which has benefits in geophysical applications with Coriolis forces. We give a normal mode analysis of the semi-discrete wave equation in one dimension which shows that the element pair is stable in dimension, and demonstrate that the element is stable with numerical integrations of the wave equation in two dimensions, an analysis of the resultant discrete Laplace operator in two and three dimensions on various meshes which shows that the element pair does not have any spurious modes. We provide convergence tests for the element pair which confirm that the element is stable since the convergence rate of the numerical solution is quadratic.
\end{abstract}

\footnotetext{
* Corresponding author.

Email addresses: colin.cotter@imperial.ac.uk (Colin J. Cotter),

d.ham@imperial.ac.uk (David A. Ham), c.pain@imperial.ac.uk (Christopher

C. Pain), sreich@math.uni-potsdam.de (Sebastian Reich).
} 


\section{Introduction}

One of the key strengths of the finite element method is the extensive choice of element types; this strength leads to endless discussion amongst practioners about the various benefits of different options. Alongside issues such as accuracy and efficiency, a key issue is that of LBB stability. This issue manifests itself in the discretisation of the wave equation (and nonlinear extensions such as the shallow-water equations and the compressible Euler equations), and also features in the discretisation of the equations of incompressible flow. Consider the wave equation written as a two-component system

$$
\begin{aligned}
\vec{u}_{t}+\nabla p & =0, \quad \vec{u}=\left(u_{1}, \ldots, u_{d}\right), \\
p_{t}+\nabla \cdot \vec{u} & =0,
\end{aligned}
$$

which, in the case of zero boundary conditions, has weak form

$$
\frac{\mathrm{d}}{\mathrm{d} t} a(\vec{u}, \vec{w})+b(p, \vec{w})=0, \quad \frac{\mathrm{d}}{\mathrm{d} t} c(\phi, p)-b(\phi, \vec{u})=0
$$

where

$$
a(\vec{u}, \vec{w})=\int_{\Omega} \vec{u} \cdot \vec{w} \mathrm{~d} V, \quad b(p, \vec{w})=\int_{\Omega} \vec{w} \cdot \nabla p \mathrm{~d} V, \quad c(\phi, p)=\int_{\Omega} \phi p \mathrm{~d} V,
$$

for suitable test functions $\vec{w}$ and $\phi$. Finite element discretisation results in the matrix form

$$
\frac{\mathrm{d}}{\mathrm{d} t} M^{u} \mathrm{u}_{i}=-C_{i} \mathrm{p}, \quad i=1, \ldots d, \quad \frac{\mathrm{d}}{\mathrm{d} t} M^{p} \mathrm{p}=\sum_{i=1}^{d} C_{i}^{T} \mathrm{u}_{i},
$$

where $C_{i}, i=1, \ldots, d$ are the finite element approximations of the Cartesian components of the gradient operator, $-\sum_{i=1}^{d} C_{i}^{T}$ is the finite element approximation to the divergence operator, $M^{u}$ and $M^{p}$ are the mass matrices associated with the finite element spaces for $u$ and $p$ respectively, and $d$ is the number of physical dimensions. By eliminating $\mathrm{u}$, we obtain the discrete wave equation

$$
M^{p} \frac{\mathrm{d}^{2}}{\mathrm{~d} t^{2}} \mathrm{p}-\sum_{i} C_{i}^{T}\left(M^{u}\right)^{-1} C_{i} \mathrm{p}=0 .
$$

If the discrete Laplace operator $\left(M^{p}\right)^{-1} \sum_{i} C_{i}^{T}\left(M^{u}\right)^{-1} C_{i}$ has null space of dimension greater than one, this results in spurious zero-energy solutions which pollute the solution after a period of time.

The null space problem also manifests itself in incompressible flow where the equations consist of a dynamical equation for $\vec{u}$ plus an incompressibility constraint which is maintained by a pressure gradient:

$$
\vec{u}_{t}+N(\vec{u})=-\nabla p+\vec{F}, \quad \nabla \cdot \vec{u}=0,
$$


where $N$ is the advective nonlinearity and $\vec{F}$ represents all other forces. In this case the spatial discretisation becomes

$$
M^{u} \frac{\mathrm{d}}{\mathrm{d} t} \mathrm{u}_{i}+\mathrm{N}_{i}(\mathrm{u})=-C_{i} \mathrm{p}+\mathrm{F}_{i}, \quad i=1, \ldots, d, \quad \sum_{i=1}^{d} C_{i}^{T} \mathrm{u}_{i}=0 .
$$

The pressure can be obtained by applying $\sum_{i} C_{i}^{T}\left(M^{u}\right)^{-1}$ to the dynamical equation for $\vec{u}$ to obtain

$$
0=\frac{\mathrm{d}}{\mathrm{d} t} \sum_{i} C_{i}^{T} \mathrm{u}_{i}=-\sum_{i} C_{i}^{T}\left(M^{u}\right)^{-1} C_{i} \mathrm{p}-\sum_{i} C_{i}^{T}\left(M^{u}\right)^{-1}\left(\mathrm{~F}_{i}-\mathrm{N}_{i}(\mathrm{u})\right),
$$

for $i=1, \ldots, d$, which can be solved for $\mathrm{p}$ (after fixing the constant component $\left.\mathrm{p}_{0}\right)$ provided that $\sum_{i} C_{i}^{T}\left(M^{u}\right)^{-1} C_{i}$ has a 1-dimensional null space containing only constant functions.

The analysis of the stability properties of finite element discretisations associated with spurious eigenvectors of $\sum_{i} C_{i}^{T}\left(M^{u}\right)^{-1} C_{i}$ was performed by Ladyzhenskaya [15], Babuska [4] and Brezzi [6]. The LBB stability condition is defined as follows:

Definition 1 For any chosen function g consider the following equation

$$
\vec{u}+\nabla p=0, \quad-\nabla \cdot \vec{u}+g=0,
$$

so that

$$
-\nabla^{2} p=g
$$

The weak form of this equation is

$$
a(\vec{u}, \vec{w})+b(p, \vec{w})=0, \quad c(\phi, g)+b(\phi, \vec{u})=0,
$$

leading to the Galerkin discretisation

$$
a\left(\vec{u}^{\delta}, \vec{w}^{\delta}\right)+b\left(p^{\delta}, \vec{w}^{\delta}\right)=0, \quad c\left(\phi^{\delta}, g^{\delta}\right)+b\left(\phi^{\delta}, \vec{u}^{\delta}\right)=0,
$$

where $\vec{u}^{\delta}, p^{\delta}$ and $g^{\delta}$ are the finite element approximations to $\vec{u}, p$, and $g$, and where $\vec{w}^{\delta}$ and $\phi^{\delta}$ are functions from the velocity and pressure Galerkin trial spaces respectively.

The Galerkin discretisation is said to be $L B B$ stable if, for any function $\phi$ from the pressure trial space, there exists $k_{\Delta x}$ such that

$$
\sup _{\vec{w}^{\delta} \in V} \frac{b\left(\phi^{\delta}, \vec{w}^{\delta}\right)}{\sqrt{a\left(\vec{w}^{\delta}, \vec{w}^{\delta}\right)}} \geq k_{\Delta x} \sqrt{c\left(\phi^{\delta}, \phi^{\delta}\right)}
$$


where $V$ is the velocity trial space, and $k_{\Delta x}$ is a positive constant which is bounded from zero as the maximum edge length $\Delta x$ in the finite element mesh tends to zero.

This is known as the inf - sup condition. LBB stability of a finite element pair is sufficient to show convergence of the solution of the Galerkin discretisation equation (4) to the solution of equation (3) at the scaling predicted by approximation theory, i.e. $\mathcal{O}\left(\Delta x^{n+1}, \Delta x^{m+1}\right)$ where $n$ and $m$ are the orders of the polynomial spaces used in the Galerkin discretisation for $\vec{u}$ and $p$ respectively, and where $\Delta x$ is the maximum edge length in the finite element mesh.

If there are spurious eigenvectors which have eigenvalues which tend to zero as $\Delta x \rightarrow 0$, these eigenvectors correspond to functions $\phi^{\delta}$ for which it is not possible to bound $b\left(\phi^{\delta}, \vec{w}^{\delta}\right)$ away from zero in that limit. As a result, a finite element discretisation is said to be LBB stable if the discrete Laplacian is free from spurious eigenvectors. In general these spurious eigenvectors consist of extra null vectors as well as "pesky modes" which have eigenvalues which tend to zero as the mesh size goes to zero. As well as inhibiting the convergence rate of the numerical solution, the spurious null vectors, which generally only occur in discretisations using structured meshes, make it impossible to invert the discrete Laplace matrix. The "pesky modes", which arise on unstructured meshes, are nearly as problematic as they lead to very large condition numbers for the discrete Laplace matrix which make iterative methods very slow to converge, as well as impeding the accuracy of the numerical solution.

Equal order finite element pairs (pairs in which the same discretisation is used for velocity and pressure) are always LBB-unstable. One way to obtain stable element pairs is to have less degrees of freedom (DOF) for $p$ than DOF for each component of $\vec{u}$. However, this is not a sufficient condition for stability and element pairs must be tested and analysed to establish their stability properties. Requiring different DOF for velocity and pressure leads to the employment of staggered grids (such as the $C$-grid in finite difference and finite volume methods) and mixed finite elements (see [13] for a discussion of mixed elements applied to the wave equation). Another frequently employed technique is to increase the number of DOF for $\vec{u}$ increased by introducing interior modes ("bubble" functions). In this paper we suggest an alternative way of increasing the DOF for $\vec{u}$ by admitting discontinuous functions whilst keeping the continuity constraint for $p$ (combinations of discontinuous and continuous functions in mixed element pairs is discussed in [13]). This often means that it is possible to increase the order of accuracy of the discretisation of $p$ (in this case to quadratic polynomials) whilst keeping the mixed element LBB stable. Our main motivation for doing this is in geophysical fluid dynamics problems in which a Coriolis term is added to the equations; these problems often have 
the system in a state of "geostrophic balance" for which

$$
\vec{\Omega} \times \vec{u} \approx-\nabla_{H} p
$$

where $\vec{\Omega}$ is the Earth's rotation vector and $\nabla_{H}$ is the horizontal gradient. For an element pair such as P1-P1, the pressure gradients are piecewise constant whilst the Coriolis force is piecewise linear and it is not possible to find a pressure field to accurately represent this balance. This leads to pressure gradient errors which pollute the solution after short times, and it becomes necessary to subtract out the balanced pressure (discretised on a higher-order element) in order for the solution to stay near to balance. For the mixed element pair discussed in this paper, the velocity field is piecewise linear whilst the pressure field is piecewise quadratic, and it will be possible to find a pressure field which represents this balance as long as the velocity field remains relatively continuous. The discontinuous velocity functions also allow one to use standard discontinuous Galerkin techniques once advection terms are introduced into the equations. In this paper we focus on investigating the LBB stability of this element pair; in other work we have analysed the geostrophic balance properties of this element pair [8] and the treatment of advection and diffusion terms in the momentum equation.

For a full treatment of LBB stability and a summary of the stability properties of a wide range of element pairs see [10]; for an analysis of element pairs applied to the linearised shallow-water equations see [20]. It is also possible to use discontinuous functions for both velocities and pressure and this leads to the class of discontinuous Galerkin methods. See [1,11] for applications of discontinuous Galerkin methods to the wave equation and [18] for applications to ocean modelling). There have been a number of methods developed to discretise the wave equation in the first-order form (1-2) using discontinuous Galerkin methods for all variables (see [1,3], for example). An application of these schemes to shallow-water systems is given in [9]. The discontinuous Galerkin method has also been applied extensively to the Maxwell equations in first-order form ([12], for example).

In section 2 we introduce the mixed discontinuous/continuous $\mathrm{P} 1_{\mathrm{D}}-\mathrm{P} 2$ element in one, two and three dimensions and show how the boundary conditions are implemented. We also give some values for the $p$ and $\vec{u}$ DOF which show the effects of making $\vec{u}$ discontinuous. In section 3 we compute the numerical dispersion relation for this element applied to the semi-discrete wave equation which shows that the element is indeed stable in one dimension. The numerical dispersion relation has a gap in the spectrum between two branches and we show that the modes from the lower frequency branch have smaller discontinuities in $\vec{u}$ with the lowest frequencies being nearly continuous. In section 4 we show eigenvalues of discrete Laplace matrices constructed on various unstructured grids in two and three dimensions which show that the element is 
stable. In section 5 we show the results of a wave equation calculation in two dimensions on an unstructured grid which illustrates the absence of spurious modes, and in section 6 we give results of convergence tests for the element pair applied to the Poisson equation which confirm LBB stability. Finally, in section 7 we give a summary of the paper and discuss other aspects of this element which may make it a good choice for ocean modelling applications.

\section{The mixed element}

In this section we describe our mixed element formulation in one, two and three dimensions.

\subsection{Weak formulation}

We start with the wave equation in the form (1-2) with boundary conditions

$$
\vec{u} \cdot \vec{n}=f \quad \text { on } \quad \partial \Omega^{N}, \quad p=g \quad \text { on } \quad \partial \Omega^{D},
$$

where $\partial \Omega^{N}$ and $\partial \Omega^{D}$ form a partition of the boundary $\partial \Omega$ of the domain $\Omega$.

To obtain our mixed finite element formulation, we multiply equation (1) by discontinuous test functions $\vec{w}$ and multiply equation (2) by continuous test functions $\phi$, then integrate over an element $E$ to obtain

$$
\begin{aligned}
\frac{\mathrm{d}}{\mathrm{d} t} \int_{E} \vec{w} \cdot \vec{u} \mathrm{~d} V & =-\int_{E} \vec{w} \cdot \nabla p \mathrm{~d} V, \\
\frac{\mathrm{d}}{\mathrm{d} t} \int_{E} \phi p \mathrm{~d} V & =-\int_{E} \phi \nabla \cdot \vec{u} \mathrm{~d} V .
\end{aligned}
$$

Integrating equation (7) by parts and making use of the boundary conditions gives

$$
\begin{aligned}
\frac{\mathrm{d}}{\mathrm{d} t} \int_{E} \vec{w} \cdot \vec{u} \mathrm{~d} V & =-\int_{E} \vec{w} \cdot \nabla p \mathrm{~d} V, \\
\frac{\mathrm{d}}{\mathrm{d} t} \int_{E} \phi p \mathrm{~d} V & =\int_{E} \nabla \phi \cdot \vec{u} \mathrm{~d} V-\int_{\partial_{E} / \partial \Omega} \vec{n} \cdot \tilde{\vec{u}} \phi \mathrm{d} S-\int_{\partial_{E} \cap \partial \Omega} f \phi \mathrm{d} S,
\end{aligned}
$$

where $\tilde{\vec{u}}$ is the value of $\vec{u}$ on the element boundary $\partial_{E}$, and $\vec{n}$ is the unit normal to $\partial_{E}$. Here $\tilde{\vec{u}}$ is determined by the particular choice of discontinuous Galerkin scheme that we use, for example the centred scheme takes the average of the values of $\vec{u}$ on either side of the boundary. 

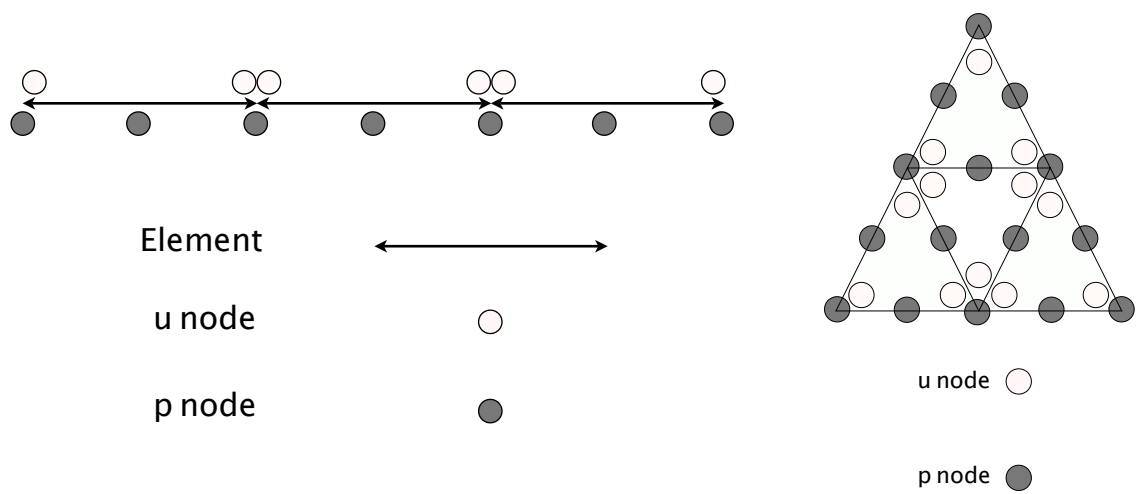

Fig. 1. Figure showing the DOF for the one-dimensional $\mathrm{P} 1_{\mathrm{D}}-\mathrm{P} 2$ element (left) and the two-dimensional $\mathrm{P} 1_{\mathrm{D}}-\mathrm{P} 2$ element (right). In one dimension, each element contains two local $\vec{u}$ DOF and three local $p$ DOF, but the global $p$ DOF are constrained to be continuous across element boundaries. In two dimensions there are three local $\vec{u}$ DOF and six local $p$ DOF.

We sum over all the elements to obtain

$$
\begin{aligned}
\frac{\mathrm{d}}{\mathrm{d} t} \int_{\Omega} \vec{w} \cdot \vec{u} \mathrm{~d} V & =-\int_{\Omega} \vec{w} \cdot \nabla p \mathrm{~d} V \\
\frac{\mathrm{d}}{\mathrm{d} t} \int_{\Omega} \phi p \mathrm{~d} V & =\int_{\Omega} \nabla \phi \cdot \vec{u} \mathrm{~d} V-\sum_{i} \int_{\Gamma_{i}}\left[\left[\vec{n}_{i} \cdot \tilde{\vec{u}} \phi\right]\right] \mathrm{d} S-\int_{\partial \Omega} f \phi \mathrm{d} S,
\end{aligned}
$$

where $\Gamma_{i}\left(i=1, \ldots, n_{\Gamma}\right)$ is the $i$ th interior element edge with normal vector $\vec{n}_{i}$, and where $[[\vec{n} \cdot \tilde{\vec{u}}]]$ is the jump in $\vec{n} \cdot \tilde{\vec{u}}$ across the element boundary. The reason that the surface integral appears as a jump term is that the test function $\phi$ is continuous across element boundaries, and therefore the trial functions from the elements on either side of each boundary appear in the same equation. If $\phi$ were discontinuous then this would not occur.

To obtain conservation we require that $[[\tilde{\vec{u}} \cdot \vec{n}]]=0$ : the same value for the flux is used on each side of the element boundary. Since $\phi$ is continuous, this means that $[[\tilde{\vec{u}} \cdot \vec{n} \phi]]=0$. This means that the integral over each $\Gamma_{i}$ vanishes and we obtain

$$
\begin{aligned}
\frac{\mathrm{d}}{\mathrm{d} t} \int_{\Omega} \vec{w} \cdot \vec{u} \mathrm{~d} V & =-\int_{\Omega} \vec{w} \cdot \nabla p \mathrm{~d} V \\
\frac{\mathrm{d}}{\mathrm{d} t} \int_{\Omega} \phi p \mathrm{~d} V & =\int_{\Omega} \nabla \phi \cdot \vec{u} \mathrm{~d} V-\int_{\partial \Omega} f \phi \mathrm{d} S .
\end{aligned}
$$




\subsection{The $P 1_{D}$-P2 element}

We then make the choice that the vector functions be discretised with discontinuous piecewise-linear $\left(\mathrm{P} 1_{\mathrm{D}}\right)$ elements and the scalar functions be discretised with continuous quadratic (P2) elements. The reason for this choice is that for all but the coarsest meshes, this element has more $\vec{u}$ DOF than $p$ DOF.

We write the global finite element expansions in the form

$$
\vec{u}_{i}(\vec{x})=\sum_{\alpha=1}^{m_{u}} \mathrm{u}_{\alpha, i} N_{\alpha}(\vec{x}), \quad p(\vec{x})=\sum_{\beta=1}^{m_{p}} \mathrm{p}_{\beta} \bar{N}_{\beta}(\vec{x}),
$$

where

$$
\mathrm{u}_{1, i}=\left[\mathrm{u}_{1, i}, \ldots, \mathrm{u}_{m_{u}, i}\right], \quad i=1, \ldots, d, \quad \mathrm{p}=\left[\mathrm{p}_{1}, \ldots, \mathrm{p}_{m_{p}}\right],
$$

and where $m_{u}, m_{p}$ are the numbers of DOF for each component of $\vec{u}$ and for $p$ respectively. This leads to the following equations:

$$
\frac{\mathrm{d}}{\mathrm{d} t} M^{u} \mathrm{u}_{i}=-C_{i} \mathrm{p}, \quad i=1, \ldots d, \quad \frac{\mathrm{d}}{\mathrm{d} t} M^{p} \mathrm{p}=\sum_{i=1}^{d} C_{i}^{T} \mathrm{u}_{i}-\mathrm{f},
$$

where

$$
\begin{aligned}
M_{\alpha, \beta}^{u} & =\int_{\Omega} N_{\alpha}(\vec{x}) N_{\beta}(\vec{x}) \mathrm{d} V(\vec{x}), \\
C_{\alpha, \beta, i} & =\int_{\Omega} N_{\alpha}(\vec{x}) \frac{\partial}{\partial x_{i}} \bar{N}_{\beta}(\vec{x}) \mathrm{d} V(\vec{x}), \\
M_{\alpha, \beta}^{p} & =\int_{\Omega} \bar{N}_{\alpha}(\vec{x}) \bar{N}_{\beta}(\vec{x}) \mathrm{d} V(\vec{x}), \\
f_{\beta} & =\int_{\partial \Omega^{N}} \bar{N}_{\beta}(\vec{x}) f(\vec{x}) \mathrm{d} S,
\end{aligned}
$$

and $d$ is the number of physical dimensions.

One of the advantages of this element choice is that the mass matrix $M^{u}$ is block diagonal (since $\vec{u}$ is discontinuous and so each global basis function is supported on only one element). This means that the discrete Laplacian is still sparse and it is not necessary to lump the mass matrix when solving the pressure equation for incompressible flow.

\subsubsection{One dimension}

In one dimension on a bounded interval of $I$ elements, there are two local DOF per element for $u$, and so there are $2 I$ global DOF as $\vec{u}$ is discontinuous. There are three local DOF per element for $p$ but there are $I-1$ global continuity 
Table 1

\begin{tabular}{|c|c|c|c|c|c|}
\hline Mesh triangles & 36 & 79 & 151 & 1586 & 15574 \\
\hline Mesh vertices & 24 & 48 & 87 & 820 & 7890 \\
\hline$\vec{u}$ DOF & 108 & 237 & 453 & 4758 & 46722 \\
\hline$p$ DOF & 85 & 176 & 326 & 2414 & 31354 \\
\hline
\end{tabular}

Table showing degrees of freedom for the $\mathrm{P} 1_{\mathrm{D}} \mathrm{P} 2$ element pair in two dimensions. The ratio of $\vec{u} \mathrm{DOF}$ to $p$ DOF appears to converge to 1.5 for large unstructured meshes.

constraints on the interfaces between each element (see figure 1). This means that there are $3 I-(I-1)=2 I+1$ global DOF for $p$, and so there is always one more $p$ DOF than $u$ DOF. However, for strong Dirichlet conditions for $p$, or periodic boundary conditions, we decrease the number of $p$ DOF and gain the potential for a stable element.

\subsubsection{Two dimensions}

In two dimensions we have $F$ triangular elements, with three local DOF per element $\vec{u}$ and six local DOF per element for $p$. There are no continuity constraints for $\vec{u}$ and so there are $3 F$ DOF (see figure 1). There is a $p$ DOF situated at each vertex and a $p$ DOF situated on each edge, and so there are $V+E p$ global DOF, where $V$ is the number of vertices and $E$ is the number of edges. Euler's formula gives $E=V+F+1$ and so there are $2 V+F+1 h$ DOF. This means that it is always possible to modify a mesh so that there are more $\vec{u}$ DOF than $p$ DOF, for example by repeatedly inserting new vertices into a triangles, breaking them into four, each time increasing $V$ by 1 and $F$ by 3. In practise, useful meshes generally satisfy $F>V$ and so this property is satisfied. Strong Dirichlet boundary conditions for $\vec{u}$ may reduce the number of $\vec{u}$ DOF below that of $p$. Table 1 gives some DOF for various unstructured Delaunay meshes in a square domain. Note that there are more velocity DOF than pressure DOF for all the meshes down to very large element sizes.

\subsubsection{Three dimensions}

In three dimensions there are four local $\vec{u} \mathrm{DOF}$ and six local $p$ DOF. As there are no continuity constraints for $\vec{u}$, there are $4 T$ global $\vec{u}$ DOF, where $T$ is the total number of tetrahedra. There is one global $p$ DOF on each vertex, and one global $p$ DOF on each edge, so there are $V+E$ global $p$ DOF. As for three dimensions, it always possible to increase $T$ relative to $V+E$ by splitting elements. Table 2 gives some DOF for sample unstructured Delaunay meshes in a cubic domain. Note that there are more velocity DOF than pressure DOF for all the meshes down to very large element sizes. 
Table 2

\begin{tabular}{|c|c|c|c|c|c|}
\hline Mesh tetrahedra & 44 & 215 & 398 & 2003 & 19140 \\
\hline Mesh vertices & 26 & 80 & 130 & 488 & 3690 \\
\hline Mesh edges & 93 & 227 & 633 & 2792 & 24165 \\
\hline$\vec{u}$ DOF & 176 & 860 & 1592 & 8012 & 77640 \\
\hline$p$ DOF & 119 & 307 & 763 & 3280 & 27855 \\
\hline
\end{tabular}

Table showing degrees of freedom for the $\mathrm{P} 1_{\mathrm{D}} \mathrm{P} 2$ element pair in three dimensions. The ratio of $\vec{u} \mathrm{DOF}$ to $p$ DOF appears to converge to 2.5 for large unstructured meshes.

\section{One-dimensional analysis}

In this section we analyse the $\mathrm{P} 1_{\mathrm{D}}-\mathrm{P} 2$ element applied to the scalar wave equation in one-dimension on a regular grid with periodic boundary conditions.

The local (elemental) mass and gradient matrices are:

$$
\bar{M}_{i j}^{u}=\int_{0}^{\Delta x} N_{i} N_{j} \mathrm{~d} x, \quad \bar{M}_{i j}^{p}=\int_{0}^{\Delta x} \bar{N}_{i} \bar{N}_{j} \mathrm{~d} x, \quad \bar{C}_{i j}=-\int_{0}^{\Delta x} N_{i} \frac{\mathrm{d}}{\mathrm{d} x} \bar{N}_{j} \mathrm{~d} x,
$$

where $\left\{N_{1}, N_{2}\right\}$ are the linear Lagrange polynomials used to represent $u$ in the element, $\left\{\bar{N}_{1}, \bar{N}_{2}, \bar{N}_{3}\right\}$ are the quadratic Lagrange polynomials used to represent $p, \bar{M}^{u}$ is the local mass matrix for $u, \bar{M}^{p}$ is the local mass matrix for $p$ and $\bar{C}$ is the local gradient matrix. These matrices are

$$
\begin{aligned}
\bar{C} & =\left(\begin{array}{lll}
-5 / 6 & 2 / 3 & 1 / 6 \\
-1 / 6 & -2 / 3 & 5 / 6
\end{array}\right), \\
\bar{M}^{u} & =\Delta x\left(\begin{array}{ll}
1 / 3 & 1 / 6 \\
1 / 6 & 1 / 3
\end{array}\right), \\
\bar{M}^{p} & =\Delta x\left(\begin{array}{ccc}
2 / 15 & 1 / 15 & -1 / 30 \\
1 / 15 & 8 / 15 & 1 / 15 \\
-1 / 30 & 1 / 15 & 2 / 15
\end{array}\right) .
\end{aligned}
$$

After assembling the equations on a regular grid with element width $\Delta x$, we obtain 


$$
\begin{aligned}
\frac{\Delta x}{6} \frac{\mathrm{d}}{\mathrm{d} t}\left(2 u_{+}^{n}+u_{-}^{n+1}\right) & =-\frac{1}{6}\left(-5 p^{n}+4 p^{n+1 / 2}+p^{n+1}\right), \\
\frac{\Delta x}{6} \frac{\mathrm{d}}{\mathrm{d} t}\left(u_{+}^{n}+2 u_{-}^{n+1}\right) & =-\frac{1}{6}\left(-p^{n}-4 p^{n+1 / 2}+5 p^{n+1}\right), \\
\frac{\Delta x}{30} \frac{\mathrm{d}}{\mathrm{d} t}\left(-p^{n-1}+2 p^{n-1 / 2}+\right. & \\
\left.8 p^{n}+2 p^{n+1 / 2}-p^{n+1}\right) & =\frac{1}{6}\left(u_{+}^{n-1}+5 u_{-}^{n}-5 u_{+}^{n}-u_{-}^{n+1}\right), \\
\frac{\Delta x}{30} \frac{\mathrm{d}}{\mathrm{d} t}\left(2 p^{n}+16 p^{n+1 / 2}+2 p^{n+1}\right) & =\frac{1}{6}\left(4 u_{+}^{n}-4 u_{-}^{n+1}\right),
\end{aligned}
$$

where $p^{n}$ is the value of $p$ at the grid point $x^{n}, p^{n+1 / 2}$ is the value of $p$ at the midpoint $x^{n+1 / 2}, u_{-}^{n}$ is the discontinuous $u$ value to the left of $x^{n}$ and $u_{+}^{n}$ is the value to the right.

We can obtain a dispersion relation for the semi-discrete system (14-17) by substituting the ansatz

$$
\begin{aligned}
& u_{+}^{n}=\hat{u}_{+} e^{i\left(k x^{n}-\omega t\right)}, u_{-}^{n}=\hat{u}_{-} e^{i\left(k x^{n}-\omega t\right)}, \\
& p^{n}=\hat{p} e^{i k\left(x^{n}-\omega t\right)}, p^{n+1 / 2}=\tilde{p} e^{i\left(k x^{n+1 / 2}-\omega t\right)} .
\end{aligned}
$$

We obtain the matrix equation

$$
\left[\begin{array}{cccc}
-2 i w & -i w e^{i \phi} & -5+e^{i \phi} & 4 e^{1 / 2 i \phi} \\
-i w & -2 i w e^{i \phi} & -1+5 e^{i \phi} & -4 e^{1 / 2 i \phi} \\
25-5 e^{-i \phi} & -25+5 e^{i \phi} & -i w(8-2 \cos \phi) & -4 i w \cos \phi / 2 \\
-20 & 20 e^{i \phi} & -2 i w\left(1+e^{i \phi}\right) & -16 i w e^{1 / 2 i \phi}
\end{array}\right]\left(\begin{array}{c}
\hat{u}^{+} \\
\hat{u}^{-} \\
\hat{p} \\
\tilde{p}
\end{array}\right)=\left(\begin{array}{l}
0 \\
0 \\
0 \\
0
\end{array}\right)
$$

where $\phi=k \Delta x$ and $w=\omega \Delta x$. After some algebraic manipulation using Maple, this yields

$$
w= \pm 2 \sqrt{\frac{26+4 \cos (\phi) \pm \sqrt{474+448 \cos (\phi)-22 \cos (2 \phi)}}{6-2 \cos \phi}} .
$$

A plot of this numerical dispersion relation is given in figure 2. The eigenvalues in the lower branch are monotonically increasing, and there is a gap in the spectrum at $k \Delta x=\pi$. The eigenvalues do not return to zero in the upper branch. The numerical dispersion relation indicates that there are no spurious modes in the discretisation and so the element is stable. Another feature is that the low frequency branch is very close to the exact dispersion relation for the wave equation.

To investigate this gap in the spectrum further, we used this solution to recover 


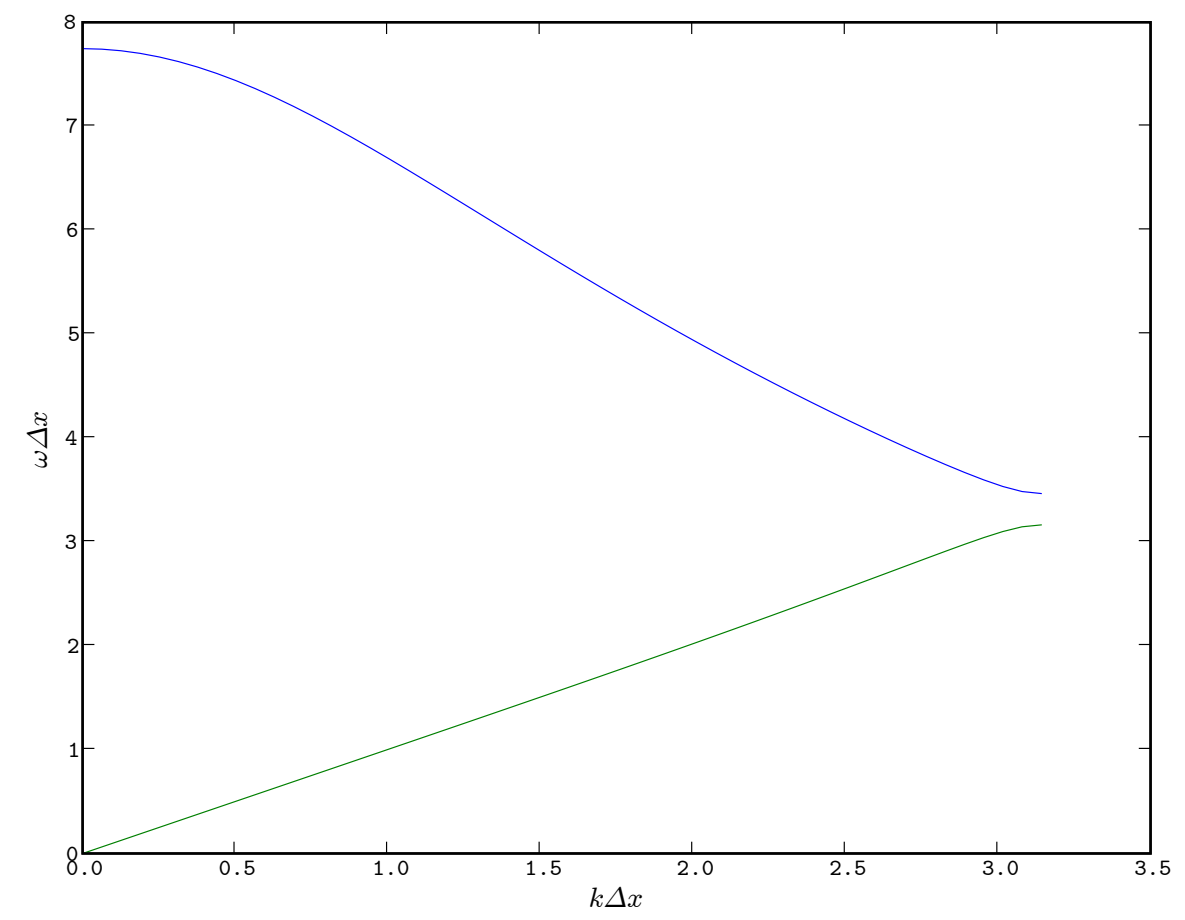

Fig. 2. Plot of the dispersion relation for the semi-discrete equations obtained from the $\mathrm{P} 1_{\mathrm{D}}-\mathrm{P} 2$ element in one dimension. The eigenspectrum has two branches, with a spectral gap separating small and large eigenvalues. The lower branch is very straight (and hence accurate).

the structure of the modes by looking at the eigenvectors of the matrix in equation (18) when $\omega$ takes these values. We normalised the eigenvectors and calculated the magnitude of the difference between $\hat{u}^{+}$and $\hat{u}^{-}$, which gives a measure of the discontinuity in each mode. Figure 3 illustrates that the level of discontinuity for modes from the lower frequency branch is much lower than for those from the higher frequency branch.

\section{Analysis of discrete Laplacian for two and three dimensional unstructured meshes}

In this section we construct the discrete Laplacian using the $\mathrm{P} 1_{\mathrm{D}}-\mathrm{P} 2$ element for unstructured meshes in two and three dimensions and check the eigenvalues for spurious modes. The meshes were produced by Triangle[21] (in two dimensions) and Tetgen[22] (in three dimensions). The matrices $C, M^{u}$ and $M^{h}$ were assembled using exact quadrature and the eigenvalues of the discrete Laplacian $\left(M^{p}\right)^{-1} \sum_{i} C_{i}^{T}\left(M^{u}\right)^{-1} C_{i}$ were computed numerically using Scientific Python. 

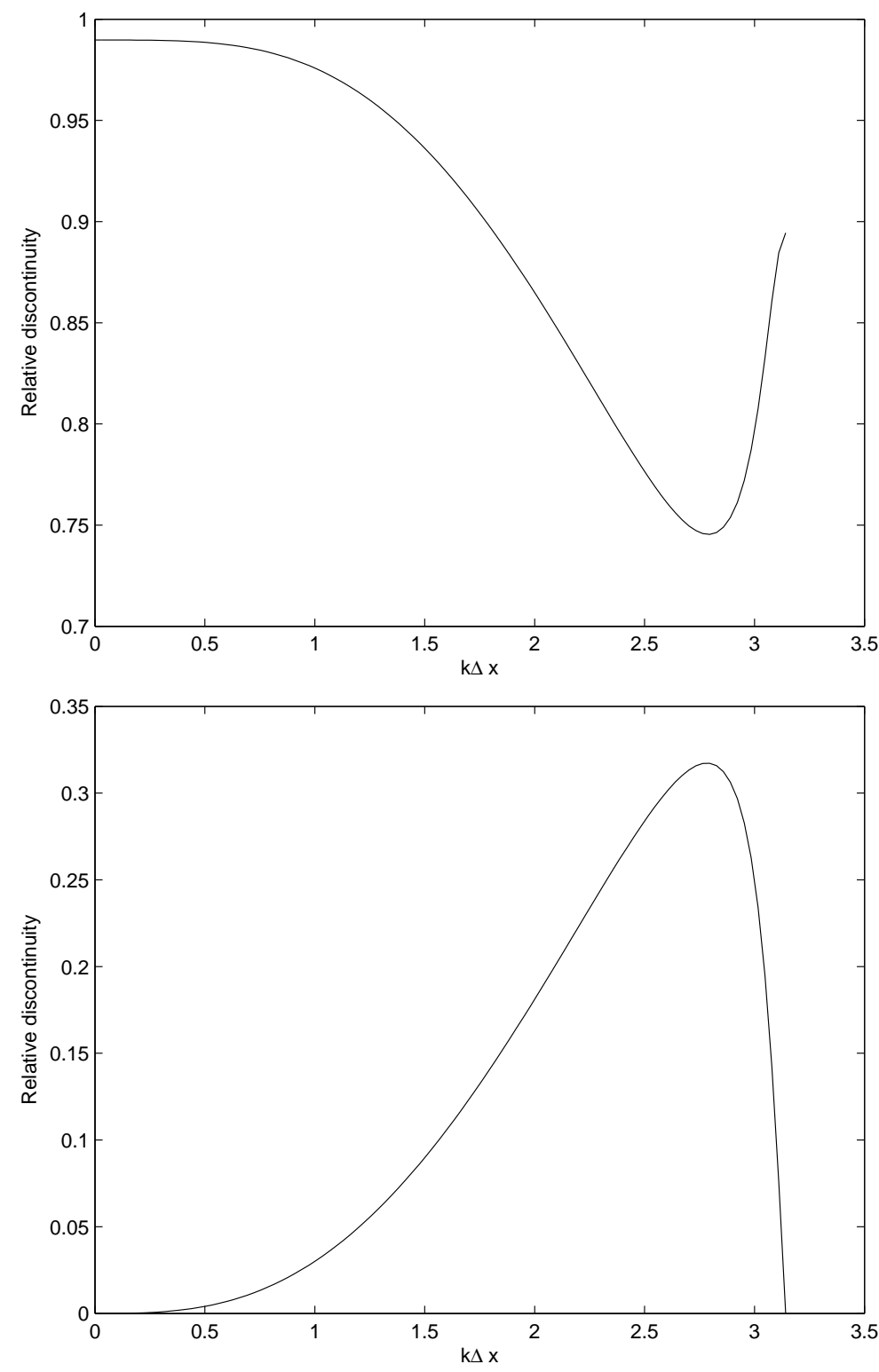

Fig. 3. Plots of the magnitude of the discontinuity in $u$ of the eigenmodes for the low (bottom plot) and high (top plot) frequency branches of the dispersion relation. The low frequency modes exhibit low levels of relative discontinuity and the high frequency modes are very discontinuous with the fastest mode being completely out of phase.

\subsection{Two dimensions}

Table 3 shows the computed eigenvalues for the discrete Laplacian obtained from the $\mathrm{P} 1_{\mathrm{D}}-\mathrm{P} 2$ element in two dimensions with Neumann boundary conditions for $\vec{u}$ and Dirichlet boundary conditions for $p$. The meshes are unstructured in a $1 \times 1$ square domain. 


\begin{tabular}{|c|c|c|}
\hline $\begin{array}{l}\text { Max } \\
\text { triangle area }\end{array}$ & $\begin{array}{l}\text { Number } \\
\text { of elements }\end{array}$ & $\begin{array}{l}\text { vector of eigenvalues } \\
\text { in increasing magnitude }\end{array}$ \\
\hline 0.1 & 14 & $\begin{array}{c}{[19.96,51.56,53.31,95.24,102.44} \\
109.80, \ldots, 555.49,632.87]\end{array}$ \\
\hline 0.05 & 36 & $\begin{array}{c}{[19.784,49.812,50.003,81.199,81.199,} \\
\ldots, 4689.754,9063.670]\end{array}$ \\
\hline 0.01 & 151 & $\begin{array}{c}{[19.741,49.375,49.379,79.104,79.104} \\
\ldots, 88388.575,210048.520]\end{array}$ \\
\hline
\end{tabular}

Table 3

Table showing eigenvalues of the discrete Laplacian obtained from the $\mathrm{P} 1_{\mathrm{D}}-\mathrm{P} 2$ element pair in two dimensions with Dirichlet boundary conditions. All the eigenvalues correspond to physical modes.

\begin{tabular}{|c|c|c|}
\hline $\begin{array}{l}\text { Max } \\
\text { triangle area }\end{array}$ & $\begin{array}{c}\text { Number } \\
\text { of elements }\end{array}$ & $\begin{array}{c}\text { vector of eigenvalues } \\
\text { in increasing magnitude }\end{array}$ \\
\hline 0.1 & 14 & $\begin{array}{l}{[0.00,9.89,9.90,19.90,} \\
41.22, \ldots, 830.0,960.9]\end{array}$ \\
\hline 0.05 & 36 & $\begin{array}{l}{[0.00,9.88,9.88,19.80,} \\
40.43, \ldots, 15870,41690]\end{array}$ \\
\hline 0.01 & 151 & $\begin{array}{l}{[0.00,9.87,9.87,19.74,} \\
39.50, \ldots, 177300,3274000]\end{array}$ \\
\hline
\end{tabular}

Table 4

Table showing eigenvalues of the discrete Laplacian obtained from the $\mathrm{P} 1_{\mathrm{D}}-\mathrm{P} 2$ element pair in two dimensions with Neumann boundary conditions. All the eigenvalues correspond to physical modes.

The Dirichlet boundary conditions for $p$ prohibit the constant $p$ solution with eigenvalue zero and so the smallest physical eigenvalue is $2 \pi^{2}$ corresponding to $p=\sin (x) \sin (y)$. It is clear from the table that there are no spurious eigenvalues (i.e. eigenvalues that scale with the mesh size) and all of the eigenvalues correspond to physical modes.

Table 4 shows the computed eigenvalues for the discrete Laplacian obtained from the $\mathrm{P} 1_{\mathrm{D}^{-}} \mathrm{P} 2$ element in two dimensions with Neumann boundary conditions for $p$ and Dirichlet boundary conditions for $\vec{u}$, on the same $1 \times 1$ domain. The Neumann boundary conditions for $p$ admit the constant $p$ solution with eigenvalue zero. The next two physical eigenfunctions are $\sin (\pi x)$ and $\sin (\pi y)$ which both have eigenvalues $\pi^{2}$. There are no spurious eigenvalues. 


\begin{tabular}{|c|c|c|}
\hline $\begin{array}{l}\text { Max } \\
\text { tetrahedral volume }\end{array}$ & $\begin{array}{c}\text { Number } \\
\text { of elements }\end{array}$ & $\begin{array}{l}\text { vector of eigenvalues } \\
\text { in increasing magnitude }\end{array}$ \\
\hline 0.1 & 44 & $\begin{array}{c}{[32.143,78.474,79.665,109.179,109.179} \\
\ldots, 465.786,599.494]\end{array}$ \\
\hline 0.01 & 215 & $\begin{array}{c}{[30.057,61.666,61.781,62.011,62.011} \\
\ldots, 4208.501,4542.808]\end{array}$ \\
\hline 0.005 & 398 & $\begin{array}{c}{[29.783,60.246,60.452,60.598,60.598} \\
\ldots, 6832.947,8060.542]\end{array}$ \\
\hline 0.004 & 487 & $\begin{array}{c}{[29.757,60.169,60.298,60.313,60.313} \\
\ldots, 8555.671,10835.590]\end{array}$ \\
\hline 0.003 & 681 & $\begin{array}{c}{[29.694,59.783,59.847,59.892,59.892} \\
\ldots, 9626.549,11173.040]\end{array}$ \\
\hline
\end{tabular}

Table 5

Table showing eigenvalues of the discrete Laplacian obtained from the $\mathrm{P} 1_{\mathrm{D}}-\mathrm{P} 2$ element pair in three dimensions with Dirichlet boundary conditions. All the eigenvalues correspond to physical modes, indicating that the element pair is stable.

\subsection{Three dimensions}

Table 5 shows the computed eigenvalues for the discrete Laplacian obtained from the $\mathrm{P} 1_{\mathrm{D}}-\mathrm{P} 2$ element in three dimensions with Neumann boundary conditions for $\vec{u}$ and Dirichlet boundary conditions for $p$. The meshes are unstructured in a $1 \times 1 \times 1$ cubic domain.

As in the two-dimensional case, the Dirichlet boundary conditions for $p$ prohibit the constant $p$ solution with eigenvalue zero and so the smallest physical eigenvalue is $3 \pi^{2}$ corresponding to $p=\sin (x) \sin (y) \sin (z)$. Table 5 shows that there are no spurious eigenvalues.

Table 6 shows the computed eigenvalues for the discrete Laplacian obtained from the $\mathrm{P} 1_{\mathrm{D}}-\mathrm{P} 2$ element in three dimensions with Neumann boundary conditions for $p$ and Dirichlet boundary conditions for $\vec{u}$. The meshes are unstructured in a $1 \times 1 \times 1$ cubic domain.

The Neumann boundary conditions for $p$ admit the constant $p$ solution with eigenvalue zero. The next three physical eigenfunctions are $\sin (\pi x), \sin (\pi y)$ and $\sin (\pi z)$ which both have eigenvalues $\pi^{2}$. There are no spurious eigenvalues. 


\begin{tabular}{|c|c|c|}
\hline $\begin{array}{l}\text { Max } \\
\text { tetrahedral volume }\end{array}$ & $\begin{array}{c}\text { Number } \\
\text { of elements }\end{array}$ & $\begin{array}{c}\text { vector of eigenvalues } \\
\text { in increasing magnitude }\end{array}$ \\
\hline 0.1 & 44 & $\begin{array}{r}{[0.00,9.93,9.93,10.06} \\
20.15, \ldots, 984,1097]\end{array}$ \\
\hline 0.01 & 215 & $\begin{array}{l}{[0.00,9.88,9.88,9.89} \\
19.83, \ldots, 5385,5931]\end{array}$ \\
\hline 0.005 & 398 & $\begin{array}{r}{[0.00,9.874,9.874,9.875} \\
19.78, \ldots, 7746,12070]\end{array}$ \\
\hline 0.004 & 487 & $\begin{array}{c}{[0.00,9.873,9.873,9.873} \\
19.78, \ldots, 10780,13010]\end{array}$ \\
\hline 0.003 & 681 & $\begin{array}{c}{[0.00,9.872,9.872,9.873} \\
\quad 19.76, \ldots, 11600,12330]\end{array}$ \\
\hline
\end{tabular}

Table 6

Table showing eigenvalues of the discrete Laplacian obtained from the $\mathrm{P} 1_{\mathrm{D}}-\mathrm{P} 2$ element pair in three dimensions with Neumann boundary conditions. All the eigenvalues correspond to physical modes, indicating that the element pair is stable.

\section{Numerical test for the wave equation}

In this section we test the $\mathrm{P} 1_{\mathrm{D}}-\mathrm{P} 2$ element as applied to the wave equation in two dimensions, with the aim of checking that spurious oscillations do not appear and that the solution remains smooth.

We discretised the equations in time using the Störmer-Verlet method given by

$$
\begin{gathered}
M^{u} \frac{\mathrm{u}_{i}^{n+1 / 2}-\mathrm{u}_{i}^{n}}{2 \Delta t}=-C_{i} \mathrm{p}^{n}, i=1, \ldots d, \\
M^{p} \frac{\mathrm{p}^{n+1}-p^{n}}{\Delta t}=\sum_{i=1}^{d} C_{i}^{T} \mathrm{u}^{n+1 / 2}, \\
M^{u} \frac{\mathrm{u}_{i}^{n+1}-\mathrm{u}_{i}^{n+1 / 2}}{2 \Delta t}=-C_{i} \mathrm{p}^{n+1}, i=1, \ldots d,
\end{gathered}
$$

This method is second-order in time, and is symplectic, one of the consequences of which is that there exists a conserved energy which is equal to the exact spatially discretised energy plus a correction of magnitude $\mathcal{O}\left(\Delta t^{2}\right)$ (see [17] for a review of the Störmer-Verlet method applied to PDEs). This means that small-scale energy will not be dissipated and it provides a good test of the spatial discretisation. As this method is explicit, there is a numerical CFL 

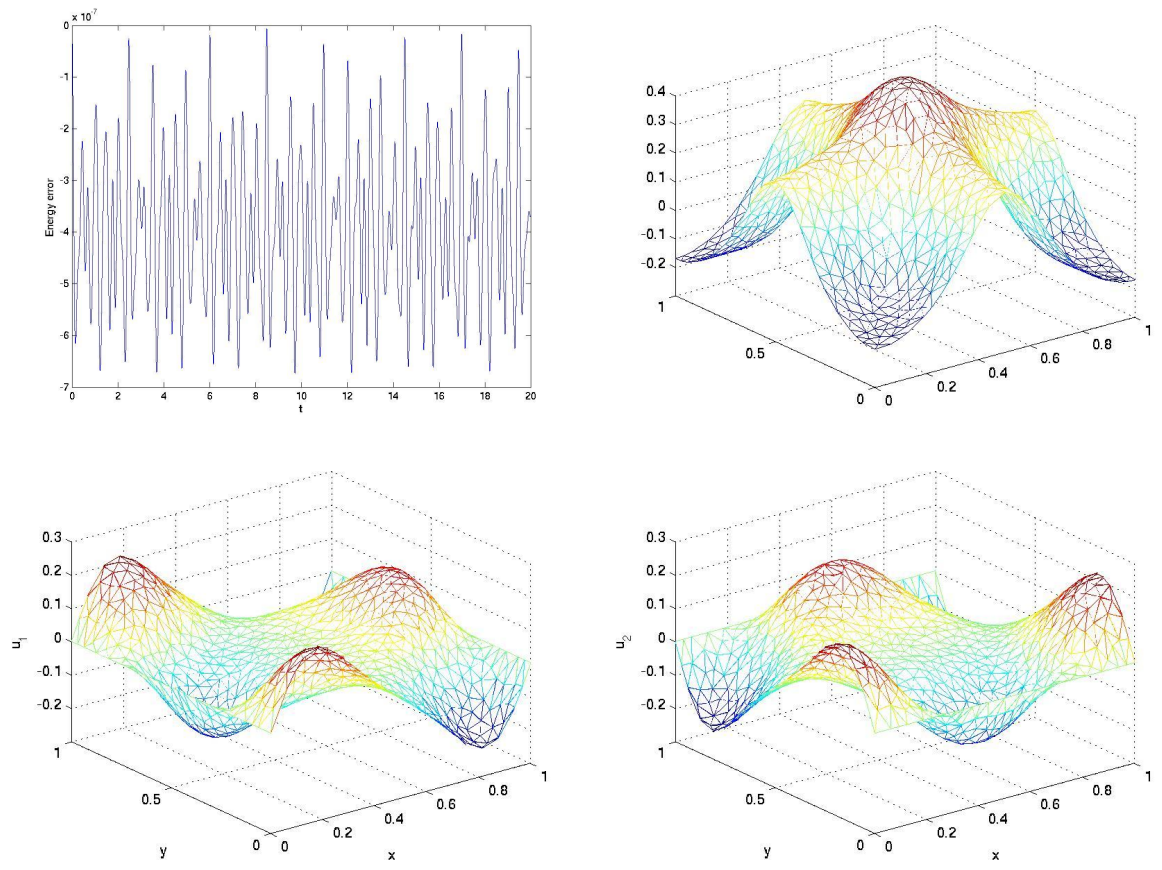

Fig. 4. Numerical results obtained from solving the 2-dimensional wave equation in a square domain of area 1 with an unstructured grid with triangular elements of typical area 0.001. The wave speed is $c=1$ and the timestep is $\Delta t=0.001$. Top-left: plot of energy error against time. Top-right: plot of $p$ at time $t=20.0$. Bottom-left and bottom-right: plots of the $x$ - and $y$-components of $\vec{u}$. These results show that the numerical solution stays smooth after a large number of timesteps, which is a good indicator that the method is stable. Although it appears from the plot that $\vec{u}$ remains almost continuous, small discontinuities are present in the solution.

condition which requires that the fastest oscillation in the system, corresponding to the largest eigenvalue of the discrete Laplacian, should be resolved in time. This discretisation still requires linear systems to be solved to obtain $\vec{u}$ and $p$ at the next time level, although the mass matrix for $\vec{u}$ is block diagonal (with one block per element).

Simulation results are given in figure 4 . These results show that the solutions remain smooth and that there are no spurious modes polluting the solution. This good behaviour arises from the stability of the $\mathrm{P} 1_{\mathrm{D}}-\mathrm{P} 2$ element.

\section{Convergence tests}

In this section we give some results of convergence tests for the $\mathrm{P} 1_{\mathrm{D}}-\mathrm{P} 2$ element pair in two and three dimensions, as well as a comparison with the $\mathrm{P} 1_{\mathrm{D}}-\mathrm{P} 1$ element pair. In these tests we formed the discrete Laplacian and used it to numerically solve the Poisson equation. 
We ran tests using the following four problems:

- $\nabla^{2} p=\sin (2 \pi x) \sin (2 \pi y)$ in $0 \leq x \leq 1,0 \leq y \leq 1$ with Dirichlet boundary conditions $p=0$ on $x=0, x=1, y=0$ and $y=1$.

- $\nabla^{2} p=\cos (2 \pi x) \cos (2 \pi y)$ in $0 \leq x \leq 1,0 \leq y \leq 1$ with Neumann boundary conditions $\vec{n} \cdot \nabla p=0$ on $x=0, x=1, y=0$ and $y=1$.

- $\nabla^{2} p=\sin (2 \pi x) \sin (2 \pi y) \sin (2 \pi z)$ in $0 \leq x \leq 1,0 \leq y \leq 1,0 \leq z \leq 1$. with Dirichlet boundary conditions $p=0$ on $x=0, x=1, y=0, y=1, z=0$ and $z=1$.

- $\nabla^{2} p=\cos (2 \pi x) \cos (2 \pi y) \cos (2 \pi z)$ in $0 \leq x \leq 1,0 \leq y \leq 1,0 \leq z \leq 1$, with Neumann boundary conditions $\vec{n} \cdot \nabla p=0$ on $x=0, x=1, y=0, y=1$, $z=0$ and $z=1$.

Figure 5 shows the results of these tests, with error plotted against maximum edge length in an unstructured mesh. The error is quadratic for both Dirichlet and Neumann boundary conditions. This error convergence rate confirms the LBB stability of the element pair since the error scaling is the same as that predicted by approximation theory (see [10], for example). The error scales quadratically since the velocity element used is linear.

Figure 6 shows the results of comparison between the $\mathrm{P} 1_{\mathrm{D}}-\mathrm{P} 2$ element pair and the $\mathrm{P} 1_{\mathrm{D}}-\mathrm{P} 1$ element pair with discontinuous linear velocity and continuous linear pressure, with error plotted against number of pressure DOF. Whilst the two element pairs have the same convergence scaling, the $\mathrm{P} 1_{\mathrm{D}}-\mathrm{P} 2$ element is more accurate for the same number of pressure DOF for sufficiently fine meshes.

\section{Summary and Discussion}

In this paper we introduced the $\mathrm{P} 1_{\mathrm{D}}-\mathrm{P} 2$ mixed element which has discontinuous velocity and continuous pressure. This choice means that the number of DOF for velocity can be increased in order to obtain a stable element. In section 2 we described the construction of the element in one, two and three dimensions and gave example values for the $\vec{u}$ and $p$ DOF. In future implementations in the three-dimensional non-hydrostatic Imperial College Ocean Model (ICOM) [19] we will investigate the relative merits of $\mathrm{P} 1_{\mathrm{D}^{-}} \mathrm{P} 2, \mathrm{P} 1_{\mathrm{D}^{-}}$ $\mathrm{P} 3, \mathrm{P} 2{ }_{\mathrm{D}}-\mathrm{P} 3$ and other combinations, including augmenting the $\vec{u}$ space with bubble functions, in practical applications.

In section 3 we gave a linear normal mode analysis for the element on a regular grid in one dimension with periodic boundary conditions which showed that the element is stable in this case. The dispersion relation is monotonically increasing with a spectral gap between the two branches, and the lower 

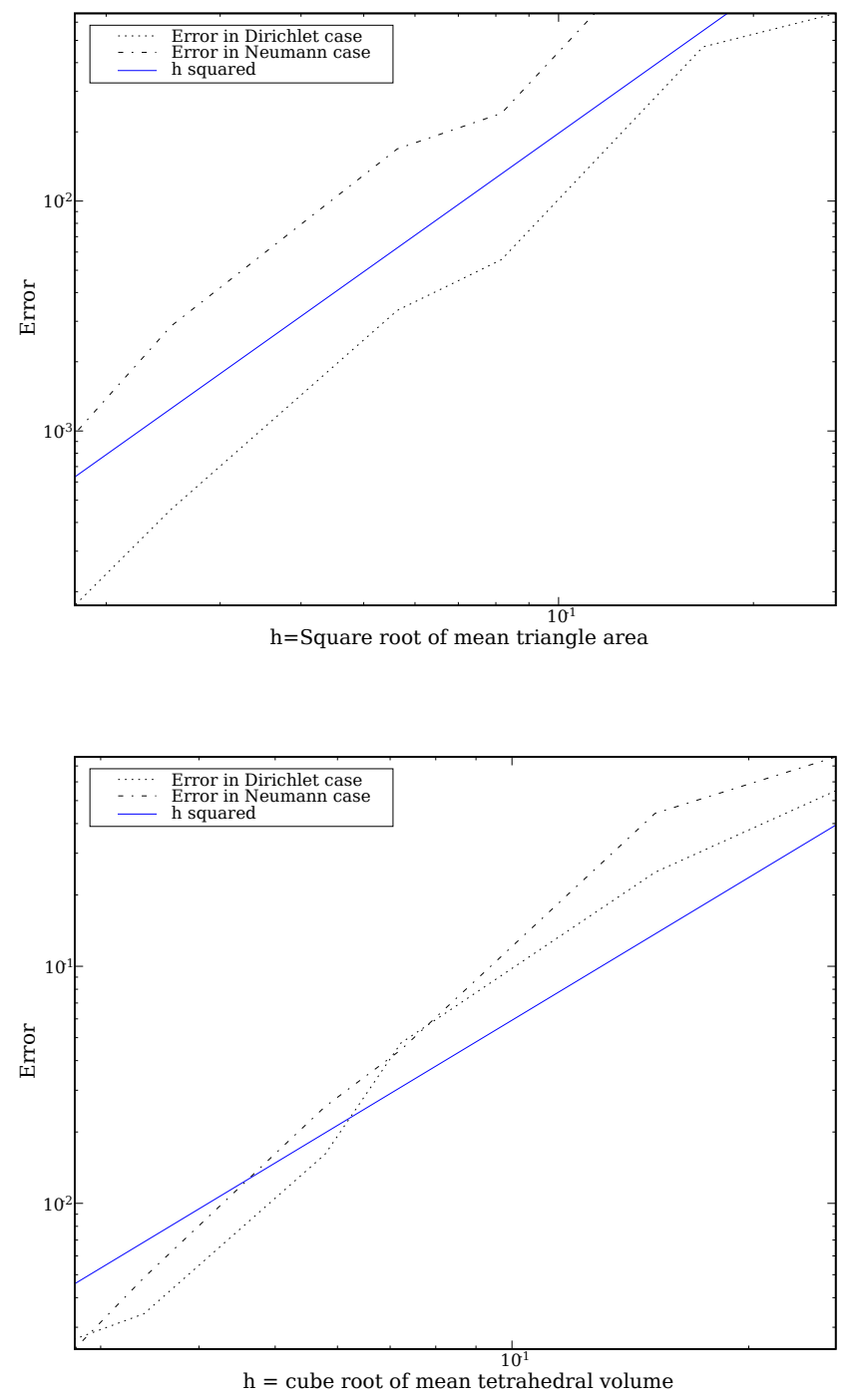

Fig. 5. Figure showing error plots for Poisson equation tests in two (upper plot) and three (lower plot) dimensions. Error is plotted against maximum edge length in the mesh. The Dirichlet condition test results are shown as dotted lines and the Neumann condition test results are shown as dash-dotted lines. A continuous line with quadratic scaling (proportional to mean edge length) is given in each plot for reference, showing that the error is quadratic in the edge length.

frequency branch has relatively continuous eigenfunctions with almost continuous eigenfunctions at the lowest frequencies.

In section 4 we presented calculations of eigenvalues of the discrete Laplace matrix obtained from unstructured meshes in two and three dimensions which demonstrated that the element is stable in these cases. In section 5 we presented results from a wave equation calculation on a two dimensional grid which demonstrated that the solutions stay smooth for relatively long time 

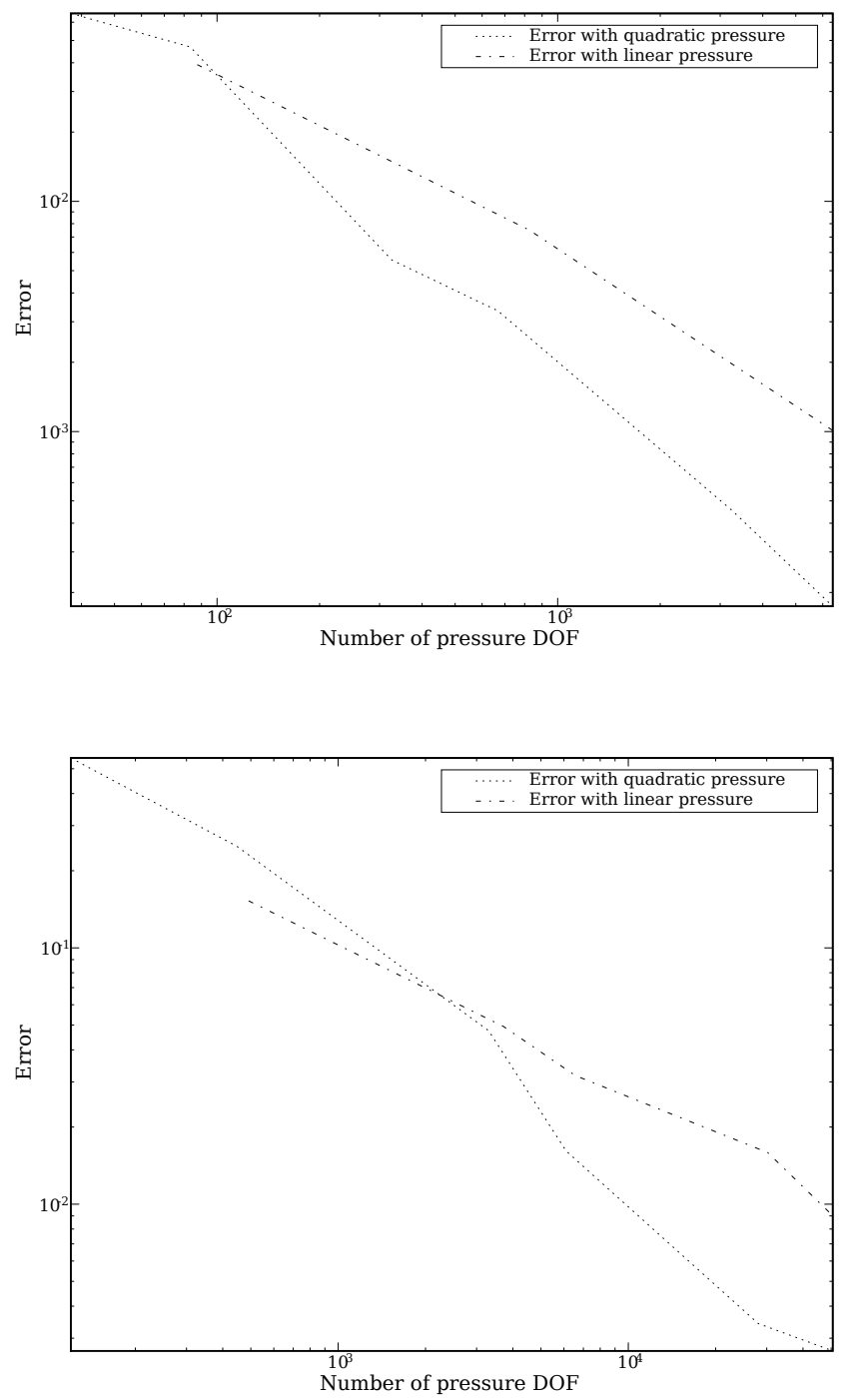

Fig. 6. Figure showing error plots for Poisson equation tests in two (upper plot) and three (lower plot) dimensions. Error is plotted against the number of pressure $\mathrm{DOF}$, for the Dirichlet condition tests only. The results using the $\mathrm{P} 1_{\mathrm{D}}-\mathrm{P} 2$ element pair are shown as dotted lines and the results using the $\mathrm{P} 1_{\mathrm{D}}-\mathrm{P} 1$ element pair are shown as dash-dotted lines. The $\mathrm{P} 1_{\mathrm{D}}-\mathrm{P} 2$ is more accurate (although with the same error scaling) than the $\mathrm{P} 1_{\mathrm{D}}-\mathrm{P} 1$ element.

intervals.

In section 6 we computed numerical solutions to the Poisson equation (which must be solved as part of the incompressible Navier-Stokes equations) obtained using the $\mathrm{P} 1_{\mathrm{D}}$ and $P 1_{D}-P 1$ element pairs. The numerical error was plotted for the $\mathrm{P} 1_{\mathrm{D}}$ element, with the optimal quadratic convergence indicating that the element is LBB-stable. The comparison with the $P 1_{D}-P 1$ element showed that although it seems sub-optimal to use piecewise-quadratic pressure when 
the same error scaling can be obtained with piecewise-linear pressure (or even piecewise-constant), the $\mathrm{P} 1_{\mathrm{D}}$ element is more accurate for the same computational cost (counting pressure DOF) and hence has the potential to be a very useful element.

This type of element with discontinuous velocity and continuous pressure has some other properties that may make it favourable for use in geophysical codes such as ICOM in which terms such as advection, diffusion and Coriolis acceleration must also be included (this can be done following a standard discontinuous Galerkin approach). The discontinuous element for velocity means that the discretisation locally conserves momentum, and allows the use of upwinding and flux-limiting to improve the treatment of advection (see, for example, [18]). As the mass matrix for $\vec{u}$ is block diagonal, the $\sum_{i} C_{i}^{T}\left(M^{u}\right)^{-1} C_{i}$ matrix remains sparse and so it is not necessary to lump the mass matrix. This makes the discretisation more accurate and reduces problems with balancing various lumped and non-lumped terms.

\section{References}

[1] M. Ainsworth, P. Monk, W. Muniz, Dispersive and dissipative properties of discontinuous Galerkin finite element methods for the second-order wave equation, J. Sci. Comput. 27 (1-3) (2006) 5-40.

[2] D. N. Arnold, An interior penalty finite element method with discontinuous elements, SIAM Journal on Numerical Analysis 19 (4) (1982) 742-760.

[3] D. N. Arnold, F. Brezzi, B. Cockburn, L. D. Marini, Unified analysis of discontinuous Galerkin methods for elliptic problems, SIAM J. Numer. Anal. 39 (5) (2001) 1749-1779.

[4] I. Babuska, Error bounds for finite element methods, Numer. Math 16 (1971) $322-333$.

[5] F. Bassi, S. Rebay, A high-order accurate discontinuous finite element method for the numerical solution of the compressible Navier-Stokes equations, J. Comp. Phys. 131.

[6] F. Brezzi, On the existence, uniqueness and approximation of saddle point problems arising from Lagrangian multiplier, Anal. Num. 8 (1974) 129-151.

[7] P. Castillo, B. Cockburn, I. Perugia, D. Schatzau, Local discontinuous Galerkin methods for elliptic problems, Communications in Numerical Methods in Engineering 18.

[8] C. J. Cotter, D. A. Ham, C. C. Pain, A mixed discontinuous/continuous finite element pair for shallow-water ocean modelling, Ocean Modelling (to appear). 
[9] C. Eskilsson, S. J. Sherwin, Discontinuous Galerkin spectral/hp element modelling of dispersive shallow water systems, J. Sci. Comput. 22 (3) (2005) $269-288$.

[10] P. M. Gresho, R. L. Sani, Incompressible Flow and the Finite Element Method, Volume 2, Isothermal Laminar Flow, Wiley, 2000.

[11] M. J. Grote, A. Schneebeli, D. Schotzau, Discontinuous Galerkin finite element method for the wave equation, SIAM Journal on Numerical Analysis 44 (6) (2006) 2408-2431.

[12] J. S. Hesthaven, T. Warburton, High-order nodal discontinuous Galerkin methods for the Maxwell eigenvalue problem, Philosophical Transactions of the Royal Society A: Mathematical, Physical and Engineering Sciences 362 (2004) 493-524.

[13] P. Joly, Topics in Computational Wave Propagation: Direct and Inverse Problems, chap. 6, Springer, 2003.

[14] G. E. M. Karniadakis, S. Sherwin, Spectral/hp Element Methods for Computational Fluid Dynamics, chap. 7, Oxford Science Publications, 2005.

[15] O. A. Ladyzhenskaya, The mathematical theory of viscous incompressible flow, Gordon and Breach, 1969.

[16] D. le Roux, A. Staniforth, C. A. Lin, Finite elements for shallow-water equation ocean models, Monthly Weather Review 126 (7) (1998) 1931-1951.

[17] B. Leimkuhler, S. Reich, Simulating Hamiltonian Dynamics, chap. 12, CUP, 2005.

[18] J. Levin, M. Iskandarani, D. Haidvogel, To continue or discontinue: Comparisons of continuous and discontinuous Galerkin formulations in a spectral element ocean model, Ocean Modelling 15 (2006) 56-70.

[19] C. Pain, M. Piggott, A. Goddard, F. Fang, G. Gorman, D. Marshall, M. Eaton, P. Power, C. de Oliveira, Three-dimensional unstructured mesh ocean modelling, Ocean Modelling 10 (2005) 5-33.

[20] D. L. Roux, A. Sène, V. Rostand, E. Hanert, On some spurious mode issues in shallow-water models using a linear algebra approach, Ocean Modelling (2005) $83-94$.

[21] J. R. Shewchuk, Triangle: engineering a 2D quality mesh generator and Delaunay triangulator, in: M. C. Lin, D. Manocha (eds.), Applied Computational Geometry: Towards Geometric Engineering, vol. 1148 of Lecture Notes in Computer Science, Springer-Verlag, 1996, pp. 203-222, from the First ACM Workshop on Applied Computational Geometry.

[22] H. Si, K. Gärtner, Meshing piecewise linear complexes by constrained Delaunay tetrahedralizations, in: Proceedings of the 14th International Meshing Roundtable, 2005. 